\title{
The Effects of Variable Viscosity, Viscous Dissipation and Chemical Reaction on Heat and Mass Transfer Flow of MHD Micropolar Fluid along a Permeable Stretching Sheet in a Non-Darcian Porous Medium
}

\author{
A. M. Salem \\ Department of Basic Science, Faculty of Computers \& Informatics, Suez Canal University, Ismailia 41611, Egypt
}

Correspondence should be addressed to A. M. Salem; azizsalem32@hotmail.com

Received 17 October 2012; Accepted 23 March 2013

Academic Editor: Mohamed Seddeek

Copyright (C) 2013 A. M. Salem. This is an open access article distributed under the Creative Commons Attribution License, which permits unrestricted use, distribution, and reproduction in any medium, provided the original work is properly cited.

\begin{abstract}
A numerical model is developed to study the effects of temperature-dependent viscosity on heat and mass transfer flow of magnetohydrodynamic(MHD) micropolar fluids with medium molecular weight along a permeable stretching surface embedded in a non-Darcian porous medium in the presence of viscous dissipation and chemical reaction. The governing boundary equations for momentum, angular momentum (microrotation), and energy and mass transfer are transformed to a set of nonlinear ordinary differential equations by using similarity solutions which are then solved numerically by shooting technique. A comparison between the analytical and the numerical solutions has been included. The effects of the various physical parameters entering into the problem on velocity, microrotation, temperature and concentration profiles are presented graphically. Finally, the effects of pertinent parameters on local skin-friction coefficient, local Nusselt number and local Sherwood number are also presented graphically. One important observation is that for some kinds of mixtures (e.g., $\mathrm{H}_{2}$, air) with light and medium molecular weight, the magnetic field and temperature-dependent viscosity effects play a significant role and should be taken into consideration as well.
\end{abstract}

\section{Introduction}

Micropolar fluids are fluids of microstructure. They represent fluids consisting of rigid, randomly oriented, or spherical particles suspended in a viscous medium, where deformation of fluids particles is ignored. The dynamics of micropolar fluids, originated from the theory of Eringen [1-3], has been a popular area of research due to its application in a number of processes that occur in industry. Such applications include polymeric fluids, real fluids with suspensions, liquid crystal, animal blood, and exotic lubricants. Extensive reviews of theory of micropolar fluids and its applications can be found in review articles by Ariman et al. $[4,5]$ and recent books by Łukaszewicz [6] and Eringen [7]. The Boundary layer concept in such fluid past a linear stretching surface has been investigated by Abo-Eldahab and El Aziz [8]. The study of heat and mass transfer flow of an electrically conducting micropolar fluid past a porous plate under the influence of a magnetic field has attracted many researchers due to its enormous applications in many engineering problems, such as MHD generators, nuclear reactors, geothermal energy extractions, and the boundary layer control in the field of aerodynamics. Eldabe et al. [9] studied the problem of heat and mass transfer in hydromagnetic flow of the nonNewtonian fluid with heat source over an accelerated surface through a porous medium. Also, Eldabe and Mohamed [10] studied numerically by using Chebyshev finite difference method, the problem of thermal-diffusion, and diffusionthermo effects on mixed free-forced convection and mass transfer boundary layer flow of non-Newtonian fluid with temperature-dependent viscosity. Bourich et al. [11] studied analytically and numerically the Soret effect on the onset of convection in a vertical porous layer subjected to uniform heat flux. Bhargava et al. [12] modeled the coupled fluid flow, heat and mass transfer phenomena over a stretching sheet with nonlinear velocity for micropolar fluid. Pal and Chatterjee Sewli [13] analyzed the steady two-dimensional 
mixed convection and mass transfer flow past a stretching surface in a micropolar fluid-saturated porous medium taking into account the Soret and Dufour effects in the presence of thermal radiation.

The combined heat and mass transfer problems with chemical reactions are of importance in many processes and therefore have received a considerable amount of attention in recent years. A reaction is said to be the first-order if the rate of reaction is directly proportional to the concentration itself. In many chemical engineering processes, a chemical reaction between a foreign mass and the fluid does occur. These processes take place in numerous industrial applications, such as the polymer production, the manufacturing of ceramics or glassware. Das et al. [14] considered the effects of first-order chemical reaction on the flow past an impulsively started infinite vertical plate with constant heat flux and mass transfer. Kandasamy et al. [15] discussed the heat and mass transfer effect along wedge with heat source and concentration in the presence of suction/injection taking into account the chemical reaction of the first-order. Pal and Chatterjee [16] studied heat and mass transfer in MHD non-Darcian flow of a micropolar fluid over a stretching sheet embedded in a porous media with nonuniform heat source and thermal radiation. The effects of chemical reaction and magnetic field on heat and mass transfer flow over a vertical isothermal cone surface in micropolar fluids with heat generation/absorption are investigated by El-kabeir and Modather [17]. Recently, Bakr [18] analyzed the effects of chemical reaction on MHD free convection and mass transfer flow of a micropolar fluid with oscillatory plate velocity and a constant heat source in a rotating frame of reference. The previous studies are based on the constant physical properties of the fluid. However, it is known that the physical properties of the fluid may change significantly with temperature [19]. The increase of temperature leads to the increase in the transport phenomena by reducing the viscosity across the momentum boundary layer and due to which the heat transfer rate at the wall is also affected. Therefore, to accurately predict the flow and heat transfer rates, it is necessary to take into account the temperature-dependent viscosity of the fluid. The effect of temperature-dependent viscosity on heat and mass transfer laminar boundary layer flow have been discussed by many authors [20-25] in various situations. They showed that when this effect was included, the flow characteristics might change substantially compared with the constant viscosity assumption. Salem [26] investigated variable viscosity and thermal conductivity effects on MHD flow and heat transfer in viscoelastic fluid over a stretching sheet. Seddeek and Salama [27] studied the effects of variable viscosity and thermal conductivity on an unsteady two-dimensional laminar flow of viscous incompressible conducting fluid past a semiinfinite vertical porous moving plate taking into account the effect of a magnetic field in the presence of variable suction.

Hence, based on the above discussion, the objective of the present study is to study the effects of variable viscosity on MHD mixed convective heat and mass transfer flow of a micropolar fluid past a porous stretching sheet in a non-Darcian porous medium with chemical reaction in the presence of viscous dissipation. The chemical reaction in this work is taken as a first-order one. The governing equations were reduced to similarity boundary layer equations using suitable transformations and then solved using the RungeKutta numerical integration with a modified version of shooting technique. Numerical results are shown graphically for the velocity, angular velocity, temperature and concentration distributions as well as the local skin friction coefficient, local Nusselt number and the local Sherwood number.

\section{Mathematical Analysis}

Consider steady two-dimensional magnetohydrodynamic heat and mass transfer flow of a viscous incompressible micropolar fluid over a continuously moving stretching surface embedded in a non-Darcian porous medium as shown in the schematic diagram in Figure 1. The surface is stretched in the $x$ direction such that the $x$ component of the velocity varies nonlinearly along it, that is, $u_{w}(x)=a x^{n}$, where $a(>0)$ is constant and $n$ is a power index. The positive $x$ coordinate is measured along the direction of motion with the slot as the origin, and the positive $y$ coordinate is measured normal to the porous plate. Here, we assume that magnetic Reynolds number of the fluid is small, so that the induced magnetic field is neglected. Also, we assume that the fluid properties are isotropic and constant, except for Newtonian fluid viscosity $\mu_{n}$ which is assumed to vary as an inverse linear function of temperature $T$ in form [28]

$$
\frac{1}{\mu_{n}}=\frac{1}{\mu_{\infty}}\left[1+\gamma\left(T-T_{\infty}\right)\right], \quad \text { that is, } \frac{1}{\mu_{n}}=A\left(T-T_{r}\right),
$$

where $A=\gamma / \mu_{\infty}$ and $T_{r}=T_{\infty}-1 / \gamma$ are constants, and their values depends on the reference state on the fluid.

Under the above assumption and using the Boussinesq approximation, the boundary layer equations for this problem can be written as follows:

$$
\begin{aligned}
& \frac{\partial u}{\partial x}+\frac{\partial v}{\partial y}=0 \\
& u \frac{\partial u}{\partial x}+v \frac{\partial u}{\partial y}= \frac{1}{\rho_{\infty}} \frac{\partial}{\partial y}\left[\left(\mu_{n}(T)+\mu_{r}\right) \frac{\partial u}{\partial y}\right] \\
&+\frac{\mu_{r}}{\rho_{\infty}} \frac{\partial \omega}{\partial y}-\left[\frac{\mu_{n}(T)+\mu_{r}}{\rho_{\infty}}\right] \frac{\varphi}{k_{1}} u-c \varphi u^{2} \\
&-\frac{\sigma B_{0}^{2}}{\rho_{\infty}} u+g \beta\left(T-T_{\infty}\right)+g \beta^{\bullet}\left(C-C_{\infty}\right)
\end{aligned}
$$




$$
\begin{gathered}
G_{1} \frac{\partial^{2} \omega}{\partial y^{2}}-2 \omega-\frac{\partial u}{\partial y}=0 \\
u \frac{\partial T}{\partial x}+v \frac{\partial T}{\partial y}=\frac{k}{\rho_{\infty} c_{p}} \frac{\partial^{2} T}{\partial y^{2}} \\
+\frac{Q_{o}}{\rho_{\infty} c_{p}}\left(T-T_{\infty}\right)+\frac{\mu_{n}(T)+\mu_{r}}{\rho_{\infty} c_{p}}\left(\frac{\partial u}{\partial y}\right)^{2} \\
u \frac{\partial C}{\partial x}+v \frac{\partial C}{\partial y}=D \frac{\partial^{2} C}{\partial y^{2}}-K\left(C-C_{\infty}\right),
\end{gathered}
$$

which is subject to the boundary conditions

$$
\begin{gathered}
u=u_{w}=a x^{n}, \quad v= \pm v_{w}, \\
T=T_{w}(x)=T_{\infty}+A x, \quad \omega=0, \\
C=C_{w} \quad \text { at } y=0, \quad u \longrightarrow 0, \\
T \longrightarrow T_{\infty}, \quad N \longrightarrow 0, \\
C \longrightarrow C_{\infty} \text { as } y \longrightarrow \infty,
\end{gathered}
$$

where $x$ and $y$ are the coordinate directions, $u, v, \omega, T$, and $C$ are the fluid velocity components in the $x$ and $y$ directions, the component of microrotation, temperature and concentration, respectively. $\mu_{n}, \mu_{r}, k_{1}, \varphi$, and $\rho_{\infty}$ are Newtonian fluid viscosity, the micropolar viscosity, the permeability of the porous medium, the porosity of the porous medium and the density of the ambient fluid. $\sigma$ is the electrical conductivity of the fluid, $B_{o}$ is the strength of applied magnetic field, $\beta$ and $\beta^{*}$ are coefficient of thermal and concentration expansions, respectively, $G_{1}$ is the microrotation constant, $c_{P}$ is the specific heat of the fluid at constant pressure, $k$ is the thermal conductivity, $Q_{o}$ is the volumetric heat generation/absorption rate, $D$ is the molecular diffusivity of the species concentration, $K$ is the rate of chemical reaction, $T_{\infty}$ is the temperature of the ambient fluid, and $v_{w}$ is the permeability of the porous surface.

The governing equations (3)-(6) can be expressed in a simpler form by introducing the following similarity transformations:

$$
\begin{gathered}
\eta=\sqrt{\frac{a(n+1)}{2 v_{\infty}}} x^{(n-1) / 2} y, \\
\omega=a x^{(3 n-1) / 2} \sqrt{\frac{a(n+1)}{2 v_{\infty}}} g(\eta), \\
\psi=x^{(n+1) / 2} \sqrt{\frac{2 a v_{\infty}}{n+1}} f(\eta), \\
u=\frac{\partial \psi}{\partial y}, \quad v=-\frac{\partial \psi}{\partial x}, \\
\theta=\frac{T-T_{\infty}}{T_{w}-T_{\infty}}, \quad \varphi=\frac{C-C_{\infty}}{C_{w}-C_{\infty}},
\end{gathered}
$$

where $\psi$ is the stream function, $\theta$ and $\varphi$ are the nondimensional temperature and concentration parameters. Substituting (8) into (3)-(6) produces the following differential equations:

$$
\begin{aligned}
&\left(\frac{\theta_{r}}{\theta_{r}-\theta}+L\right) f^{\prime \prime \prime}+\left(\frac{\theta_{r} \theta^{\prime}}{\left(\theta_{r}-\theta\right)^{2}}+f\right) f^{\prime \prime}-2\left(\frac{n+a_{1}}{n+1}\right) f^{\prime 2}+L g^{\prime} \\
&- {\left[\frac{2}{\mathrm{Da}(n+1)}\left(\frac{\theta_{r}}{\theta_{r}-\theta}+L\right)+\frac{2 M}{n+1}\right] f^{\prime} } \\
&+ \frac{2 \lambda}{n+1}\left(\theta+N \varphi^{\prime}\right)=0, \\
& G(n+1) g^{\prime \prime}-2\left(g+f^{\prime \prime}\right)=0, \\
& \theta^{\prime \prime}+\operatorname{Pr}_{\infty}\left[\frac{2}{n+1} E \theta+\left(\frac{\theta_{r}}{\theta_{r}-\theta}+L\right) \mathrm{Ec} f^{\prime \prime 2}\right]=0, \\
& \varphi^{\prime \prime}+\operatorname{Sc}\left(f \varphi^{\prime}-\frac{2 \Delta}{n+1} \varphi\right)=0,
\end{aligned}
$$

and the boundary condition (7) becomes

$$
\begin{gathered}
f(\eta)= \pm F_{w}, \quad f^{\prime}(\eta)=1, \\
\theta(\eta)=1, \quad g(\eta)=0, \quad \varphi(\eta)=1 \quad \text { at } \eta=0, \\
F^{\prime}(\eta) \longrightarrow 0, \quad \theta(\eta) \longrightarrow 0, \\
\varphi(\eta) \longrightarrow 0, \quad g(\eta) \longrightarrow 0 \quad \text { as } \eta \longrightarrow \infty,
\end{gathered}
$$

where $\theta_{r}=-1 / \gamma\left(T_{w}-T_{\infty}\right)$ is the variable viscosity parameter, $\alpha_{1}=c \varphi x$ is the local inertia coefficient parameter, $L=\mu_{r} / \mu_{\infty}$ is the vortex viscosity parameter, $\mathrm{Da}^{-1}=\varphi v_{\infty} / k_{1} a x^{n-1}$ is the inverse Darcy number, $M=\sigma B_{0}^{2} / \rho_{\infty} a x^{n-1}$ is the local magnetic field parameter, $\lambda=\mathrm{Gr}_{x} / \mathrm{Re}_{x}^{2}$ is the buoyancy force parameter, $N=\beta^{*}\left(C_{w}-C_{\infty}\right) / \beta\left(T_{w}-T_{\infty}\right)$ is the buoyancy ratio parameter, $G=G_{1} a / v_{\infty} x^{1-n}$ is the microrotation parameter, $\operatorname{Pr}=\left(1-\theta / \theta_{r}\right)^{-1} \operatorname{Pr}_{\infty}$ is the Prandtl number and $\operatorname{Pr}_{\infty}=\mu_{\infty} c_{p} / k_{\infty}$ is the ambient Prandtl number, $E=Q_{0} x^{1-n} / \rho_{\infty} c_{p} a$ is the local heat generation/absorption parameter, Ec $=a^{2} x^{2 n} / c_{p}\left(T_{w}-T_{\infty}\right)$ is the local Eckert number (characterizing viscous dissipation), $\mathrm{Sc}=v_{\infty} / D$ is the Schmidt number, $\Delta=K / a x^{n-1}$ is the chemical reaction parameter, and $F_{w}= \pm \sqrt{2} v_{w} x^{(1-n) / 2} / \sqrt{\nu_{\infty} a(n+1)}$ is the suction or injection parameter. Here $F_{w}$ is positive for suction and negative for injection.

In the above equations, a prime denotes differentiation with respect to $\eta$. For the limiting case $\theta_{r} \rightarrow \infty$ (uniform viscosity), $L=0$ (Newtonian fluid), $n=1$ (linear surface velocity), $\lambda=0$ (without buoyancy force), (9), together with 
the boundary conditions $f(0)=F_{w}, f^{\prime}(0)=1$ and $f^{\prime}(\infty)=$ 0 , has an exact closed form solution in the form

$$
\begin{gathered}
f(\eta)=\frac{1}{Z}\left(1-\exp \left(-\frac{1}{\eta} Z\right)+\frac{1}{2} F_{w} Z\right), \\
Z=F_{w}+2 \sqrt{1+\left(M+\mathrm{Da}^{-1}\right)+\frac{F_{w}^{2}}{4}} .
\end{gathered}
$$

The physical quantities of interest are the skin friction coefficient, the Nusselt number and the Sherwood number. These are defined by

$$
\begin{gathered}
c_{f}=\frac{2 \tau_{w}}{\rho_{\infty} u_{w}^{2}}, \quad \mathrm{Nu}_{x}=\frac{x q_{w}}{k\left(T_{w}-T_{\infty}\right)}, \\
\mathrm{Sh}=\frac{x m_{w}}{\rho_{\infty} D\left(C_{w}-C_{\infty}\right)},
\end{gathered}
$$

where

$$
\begin{gathered}
\tau_{w}=\left[\left(\mu_{n}(T)+\mu_{r}\right) \frac{\partial u}{\partial y}+\mu_{r} \omega\right]_{y=0}, \\
q_{w}=-k\left[\frac{\partial T}{\partial y}\right]_{y=0}, \\
m_{w}=-\rho_{\infty} D\left(\frac{\partial u}{\partial y}\right)_{y=0}
\end{gathered}
$$

are shear stress, surface heat flux, and surface mass flux, respectively. Using the new similarity variables in (8) gives

$$
\begin{gathered}
c_{f} \sqrt{\operatorname{Re}_{x}}=\sqrt{2(n+1)}\left[\left(\frac{\theta_{r}}{\theta(\theta)-\theta_{r}}+L\right) f^{\prime \prime}(0)+\operatorname{Lg}(0)\right], \\
\operatorname{NuRe}_{x}^{-1 / 2}=-\sqrt{\frac{n+1}{2}} \theta^{\prime}(0), \\
\operatorname{Sh~Re}_{x}^{-1 / 2}=-\sqrt{\frac{n+1}{2}} \varphi^{\prime}(0),
\end{gathered}
$$

where $\operatorname{Re}_{x}=u_{w} x / \nu_{\infty}$ is the local Reynolds number.

\section{Results and Discussion}

The set of nonlinear ordinary differential equations (9)(12) with the boundary conditions (13) have been solved by means of fourth-order Runge-Kutta method with systematic estimates of $F^{\prime \prime}(0), g^{\prime}(0), \theta^{\prime}(0)$, and $\varphi^{\prime}(0)$ being made by using the shooting technique. In the present calculations, the step sizes of $\Delta \eta=0.01$ and $\eta_{\max }=10$ were found to be satisfactory in obtaining sufficient accuracy within a tolerance of less than $10^{-7}$ in nearly all cases. In order to assess the accuracy of the present numerical method, we compared our numerical results obtained for the dimensionless stream function and its derivative taking into account that $L=\lambda=0$, $n=1$, and $\theta_{r} \rightarrow \infty$ in (9) with those obtained analytically. The numerical and the analytical values of $f(\eta)$ and $f^{\prime}(\eta)$ for

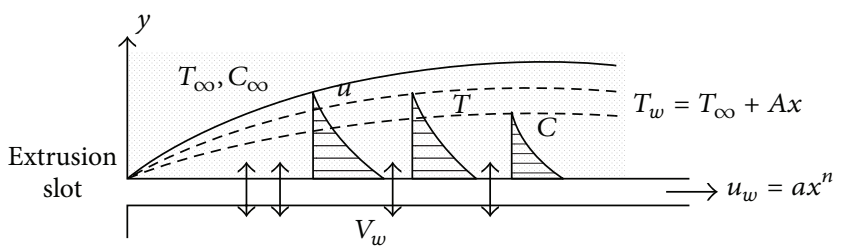

FIGURE 1: Schematic diagram of flow induced by a power-law stretched surface.

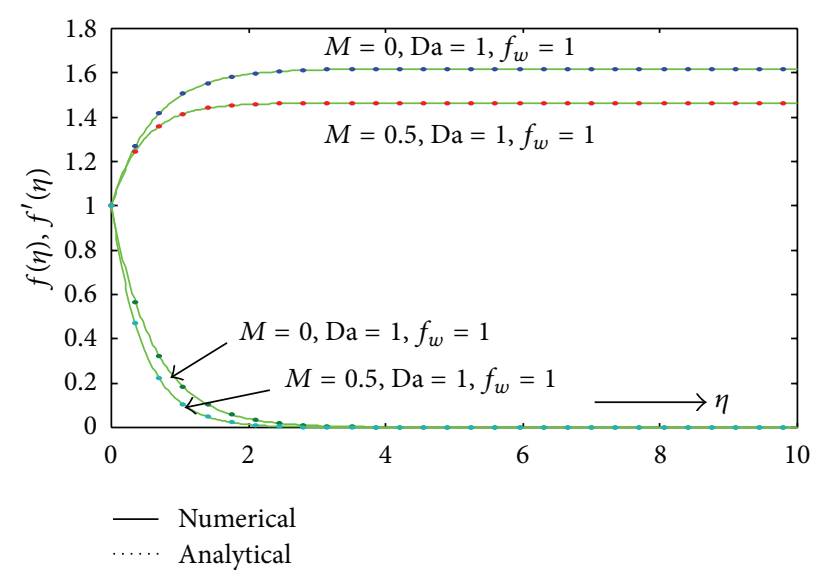

FIGURE 2: Comparison of the exact solution and numerical solution for various values of $M$.

some values of the magnetic field parameter $M$ and porosity parameter Da are shown in Figure 2. The numerical values of $f(\eta)$ and $f^{\prime}(\eta)$ are in good agreement with the obtained analytical values.

In order to obtain some physical insight of the problem, numerical results are displayed with the help of graphical illustrations. A representative set of results is shown in Figures 3-22. Numerical calculations have been carried out for different values of physical parameters such as the fluid viscosity parameter $\theta_{r}$, the viscous dissipation parameter Ec, the magnetic field parameter $M$, the coupling parameter $L$, the porosity parameter $\mathrm{Da}$, the buoyancy force parameter $\lambda$, the chemical reaction parameter $\Delta$, the suction or injection parameter $F_{w}$, and the Schmidt number Sc. In the graphs provided, the dimensionless velocity, microrotation (angular velocity), temperature and concentration profiles are computed for fixed value of the Prandtl number $\mathrm{Pr}$, the inertia coefficient parameter $\alpha_{1}$, the buoyancy ratio parameter $N$, the heat generation parameter $E$, and the microrotation parameter $G$ which are taken as $0.71,0.5,0.5,0.5$, and 2, respectively. The values of Schmidt number are chosen for hydrogen $(\mathrm{Sc}=0.22)$, water vapor $(\mathrm{Sc}=0.6)$ and ammonia $\left(S_{c}=0.78\right)$, which represent diffusion chemical species of the most common interest in air at $20^{\circ} \mathrm{C}$ and one atmospheric pressure [29].

The effects of the variable viscosity parameter $\theta_{r}$ and the magnetic field parameter $M$ on the velocity, microrotation, temperature and concentration profiles are shown in Figures 3,4 , and 5. It is observed from these figures that the velocity, microrotation, and temperature profiles of the micropolar 


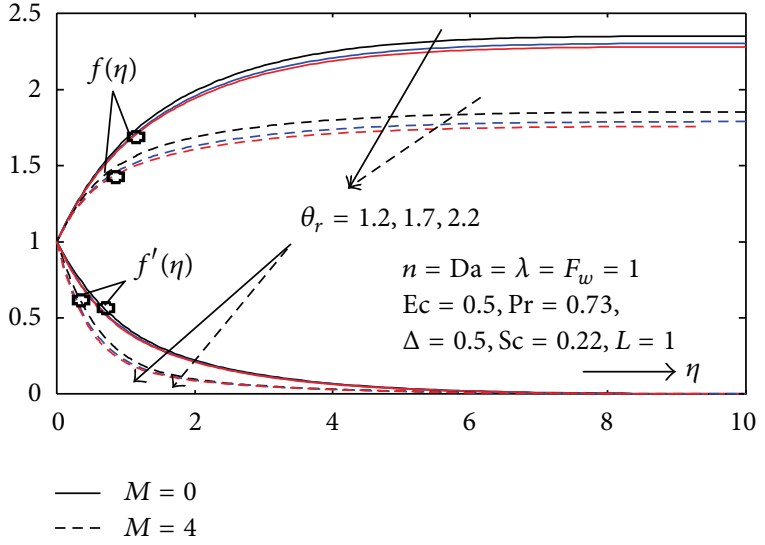

Figure 3: Velocity profile for different values of $\theta_{r}$ and $M$.

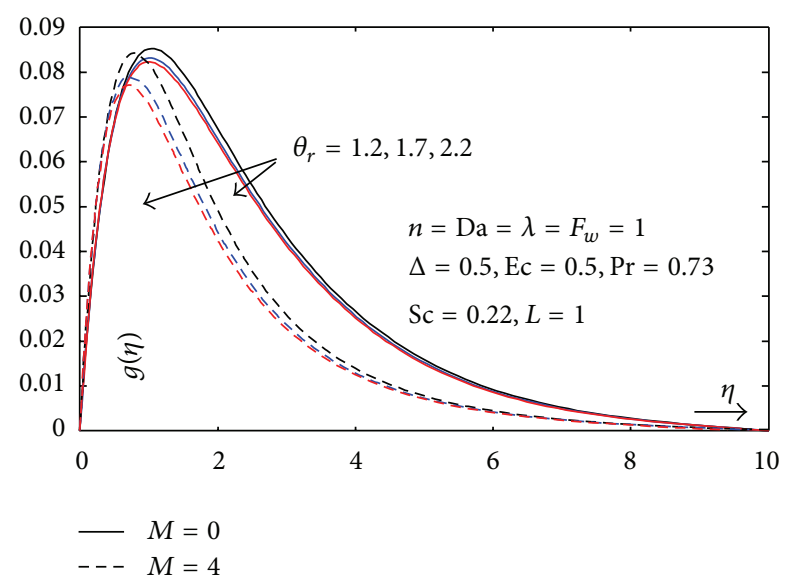

Figure 4: Angular velocity profile for different values of $\theta_{r}$ and $M$.

fluid decrease, whereas the concentration of the micropolar fluid increases as $\theta_{r}$ increases in the absence/presence of the magnetic field. In the case of magnetic field $(M \neq 0)$, varying the temperature-dependent viscosity becomes more effective on the velocity, microrotation, and temperature fields than in the nonmagnetic case $(M=0)$. Further, the temperaturedependent viscosity has only very slight influence on the concentration profiles whenever no magnetic field is applied, while the increase in concentration due to variable viscosity is recognized at a certain nonzero value of $M$.

Figures $6-8$, respectively, show the velocity, microrotation, temperature and concentration profiles for different values of Darcy number $\mathrm{Da}$ in the absence and presence of the Eckert number Ec. The presence of a porous medium in the flow presents the resistance to the flow, and in the limiting case when $\mathrm{Da} \rightarrow \infty$ value, the porosity disappears. Therefore, as the inverse Darcy number $\mathrm{Da}^{-1}$ increases, the resistance due to porous medium increases and the velocity profiles decrease as shown in Figure 6. Also, we see from this figure that the velocity increases with increasing viscous dissipation parameter Ec. This effect is more pronounced in the case of a purely fluid region (infinite $\mathrm{Da}$ ) than in the case of flow through a porous medium. Figure 7 shows

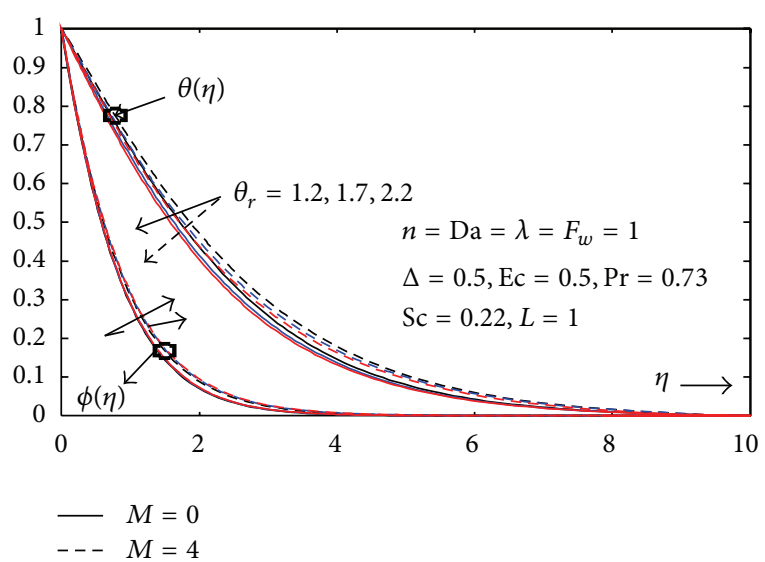

Figure 5: Temperature and profile different values of $\theta_{r}$ and $M$.

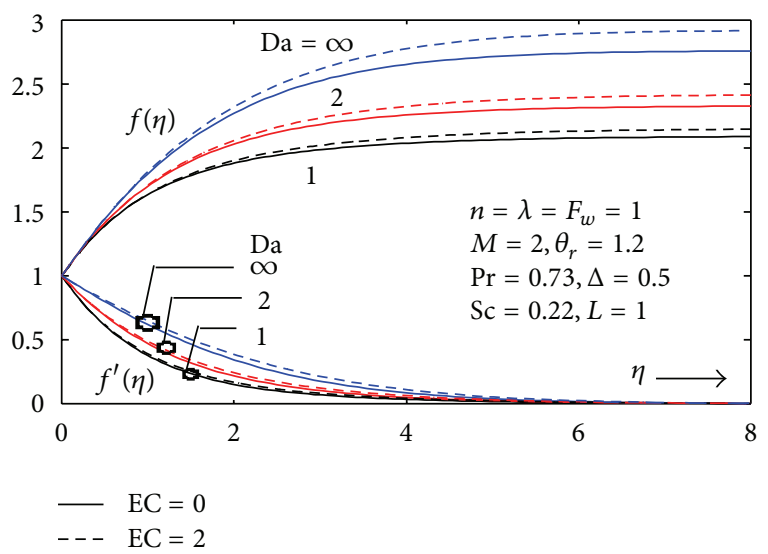

Figure 6: Velocity profile for different values of Da and Ec.

that the microrotation velocity decreases with an increase in the inverse Darcy number which means that the presence of porous medium decelerates the rotary motions of the microelements near the surface, but the reverse happens away from the surface. Figure 8 shows that the temperature and concentration distributions of the fluid increase due to increase in the inverse Darcy number $\mathrm{Da}^{-1}$. In the presence of viscous dissipation, $\mathrm{Ec} \neq 0$, the effect of $\mathrm{Da}^{-1}$ is to increase the temperature more than in the case of $\mathrm{Ec}=0$ as a result of viscous dissipation effect which acts as a heat source. Also, the viscous dissipation parameter has only very slight influence on the concentration profiles in the case of a highly porous medium.

Figures 9-12 depict the influence of the chemical reaction parameter $\Delta$ with different values of Sc on the behavior of the velocity, microrotation, temperature and concentration profiles. Increasing the chemical reaction parameter $\Delta$ produces a decrease in the species concentration for both hydrogen and ammonia. This is due to the fact that destructive chemical reaction reduces the solutal boundary layer thickness. This, in turn, causes the concentration buoyancy effects to decrease as $\Delta$ increases. Consequently, less flow is induced along the surface resulting in decreases in the fluid velocity and slight increases in the temperature profiles for both $\mathrm{Sc}=0.22$ and 


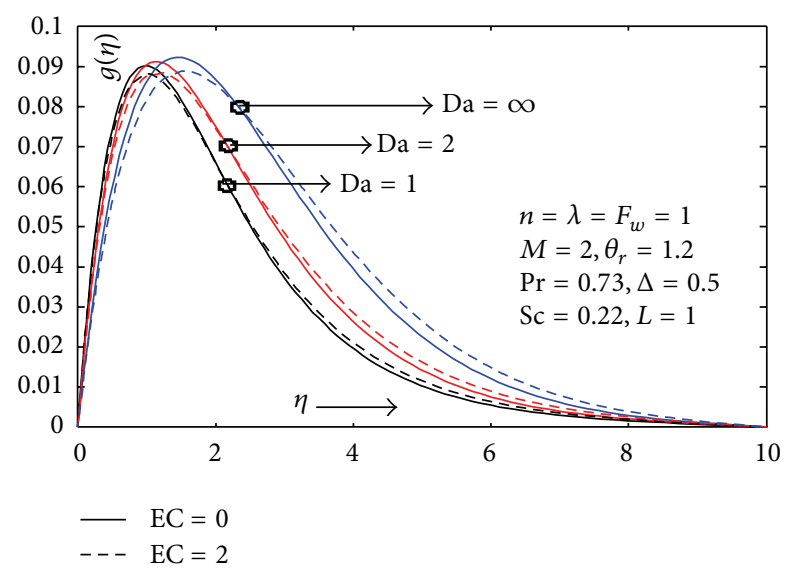

FIgURE 7: Angular velocity profile for different values of Da and Ec.

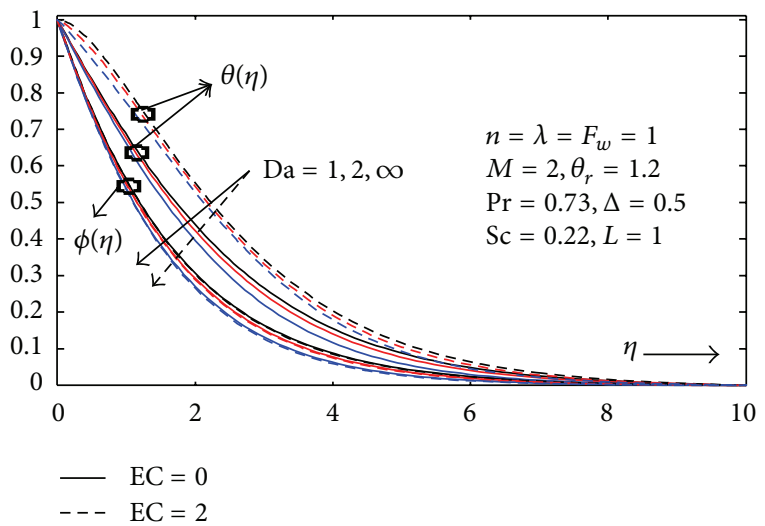

FIGURE 8: Temperature and concentration profile for different values of $\mathrm{Da}$ and $\mathrm{Ec}$.

$\mathrm{Sc}=0.78$. In addition, increasing the Schmidt number Sc leads to decreases in the concentration profiles. This result can be explained by the fact that an increase in the value of $\operatorname{Sc}\left(v_{\infty} / D\right)$ for fixed value of $v_{\infty}$ means a decrease in the molecular diffusivity $D$. Hence, concentration of species is higher for lighter particles $(\mathrm{Sc}=0.22)$ and lower for heavier particles $(\mathrm{Sc}=0.78)$. This behavior is clear from Figure 11. Figure 10 shows that with increasing $\Delta$ and Sc, the microrotation profiles increase near the porous plate, while the situation is reversed far away from the porous plate; that is, the microrotation profiles increase as $\Delta$ and Sc increase. Also, we noticed that, the effect of $\Delta$ on the microrotation profiles in the case of micropolar fluid with small Schmidt number is stronger than its effect in the case of micropolar fluid with large Schmidt number.

The variation of the velocity, temperature and concentration profiles are shown in Figures 13, 14, and 15 for various values of the suction/blowing parameter $F_{w}$ and microrotation parameter $L$. Here $F_{w}>0$ corresponding to suction and $F_{w}<$ 0 corresponding to injection at the plate. It is clear that for all values of suction/blowing parameter $F_{w}$, the temperature and concentration profiles within the boundary layer increase, while the velocity components decrease as $L$ increases (the

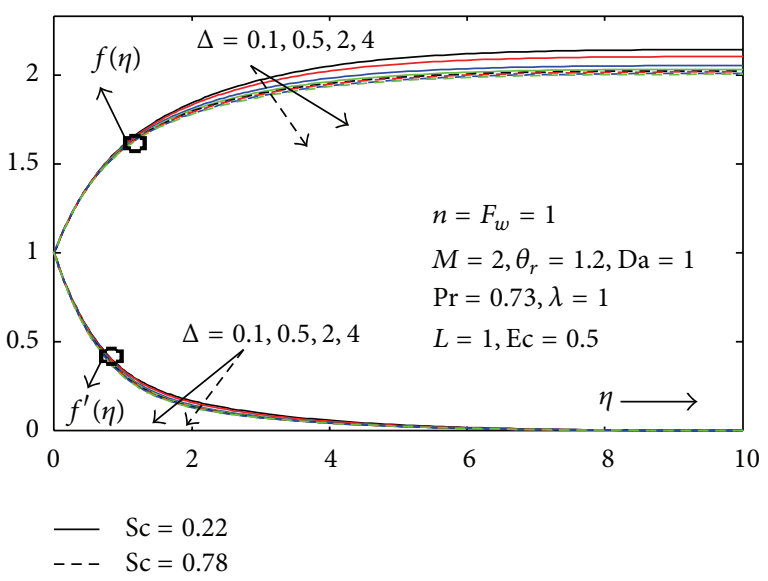

FIGURE 9: Velocity profile for different values of $\Delta$ and Sc.

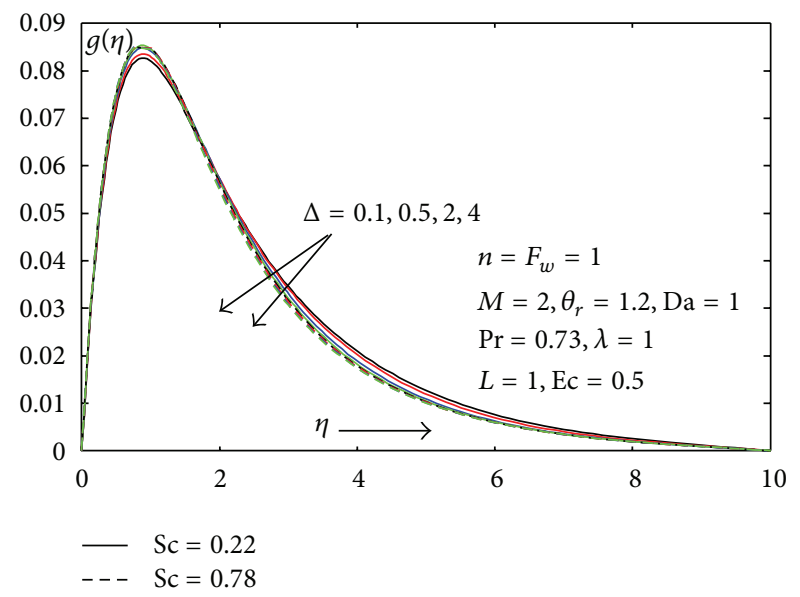

FIgURE 10: Angular velocity profile for different values of $\Delta$ and Sc.

microrotation increases). The reason for this trend is as follows: as the value of the microrotation parameter increases, the coupling between (9), (10), and (12) increases causing a whole reduction in the fluid velocity, and consequently, heat is expected to increase. Figure 16 displays the influence of the coupling parameter $L$ and suction/injection parameter $F_{w}$ on the microrotation profiles. It is seen from this figure that increasing values of the microrotation parameter $L$ result in increasing the microrotation profiles near the plate but a reverse process has occurred as one moves away from the porous plate. From these figures it is observed that the effect of microrotation parameter $L$ on the velocity, temperature, and concentration is more pronounced in the case of injection than in the case of the impermeable plate $\left(F_{w}=0\right)$ and $\operatorname{suction}\left(F_{w}=1\right)$.

The combined effect of the variable viscosity parameter $\theta_{r}$ and buoyancy force parameter $\lambda$ on the local skin-friction coefficient $c_{f} R_{x}^{1 / 2}$, the local Nusselt number $\mathrm{Nu}_{x} R_{x}^{-1 / 2}$, and the local Sherwood number $\operatorname{Sh} R_{x}^{-1 / 2}$ are shown in Figures 17,18 , and 19. In the absence of viscous dissipation, it is observed that both the local skin-friction coefficient and local Sherwood number decrease with increase of variable 


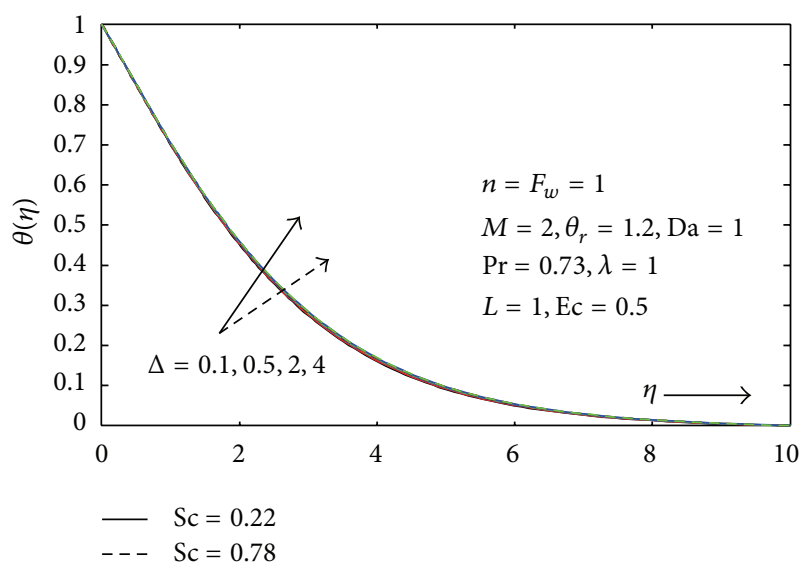

Figure 11: Temperature profile for different values of $\Delta$ and Sc.

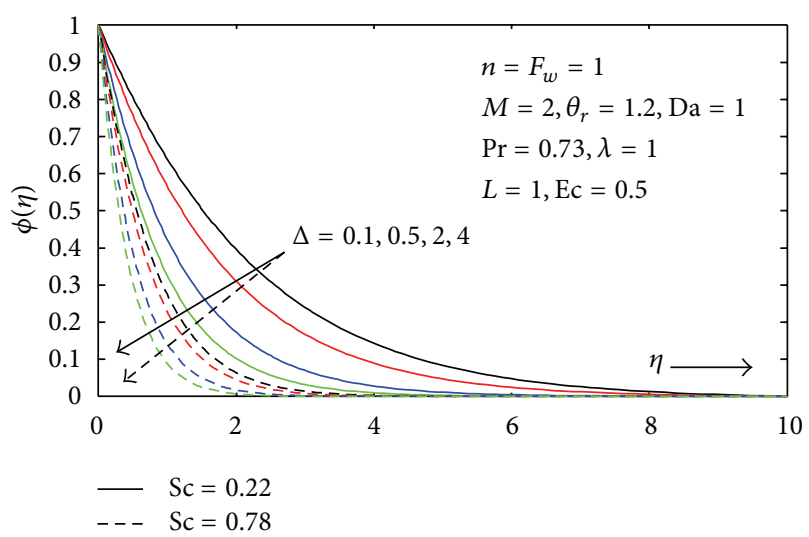

FIGURE 12: Concentration profile for values of $\Delta$ and Sc.

viscosity parameter $\theta_{r}$, whereas the local Nusselt number increases with increase of $\theta_{r}$. In the presence of viscous dissipation, the effect of $\theta_{r}$ is to increase the rate of heat transfer near the surface and decrease the same significantly away from the surface. This reduction of heat transfer away from the boundary would be more for large values of buoyancy force parameter $\lambda$. Also, for $\mathrm{Ec}=2$ the values of skin friction coefficient and Nusselt number are smaller than $\mathrm{Ec}=0$, while the values of Sherwood number are higher for $\mathrm{Ec}=2$ than $\mathrm{Ec}=0$. In addition, the effect of Ec is more pronounced on the local Nusselt number than on the local skin-friction coefficient and the local Sherwood number, because Ec occurs explicitly in (11) which represents the equation for dimensionless temperature.

The effects of the coupling parameter $L$ and the Schmidt number Sc on the local skin-friction coefficient, the local Nusselt number, and the local Sherwood number are shown in Figures 20, 21, and 22, respectively. It is observed that for all values of $\lambda$, the local skin-friction coefficient and the local Nusselt number decrease as the Schmidt number Sc increases. Also, as the value of the coupling parameter increases, the coupling between equations of momentum and energy increases causing the local skin-friction coefficient to increase while the wall heat transfer is greatly decreased. This

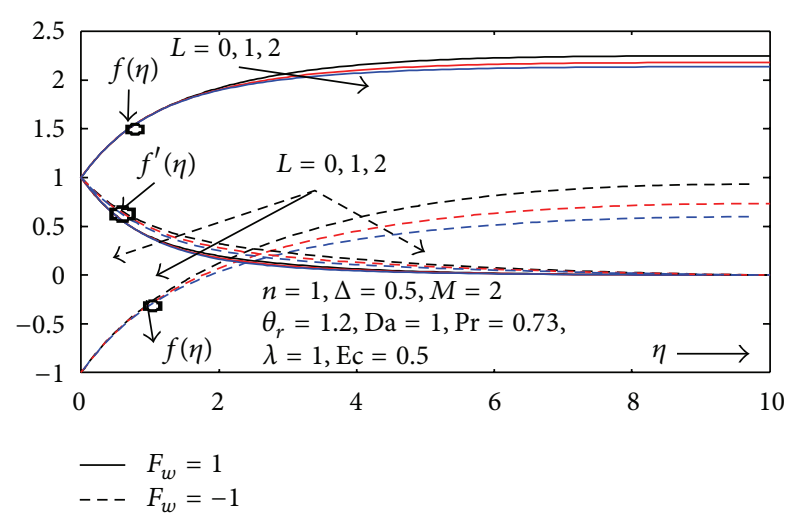

FIGURE 13: Velocity profile for different values of $F_{w}$ and $L$.

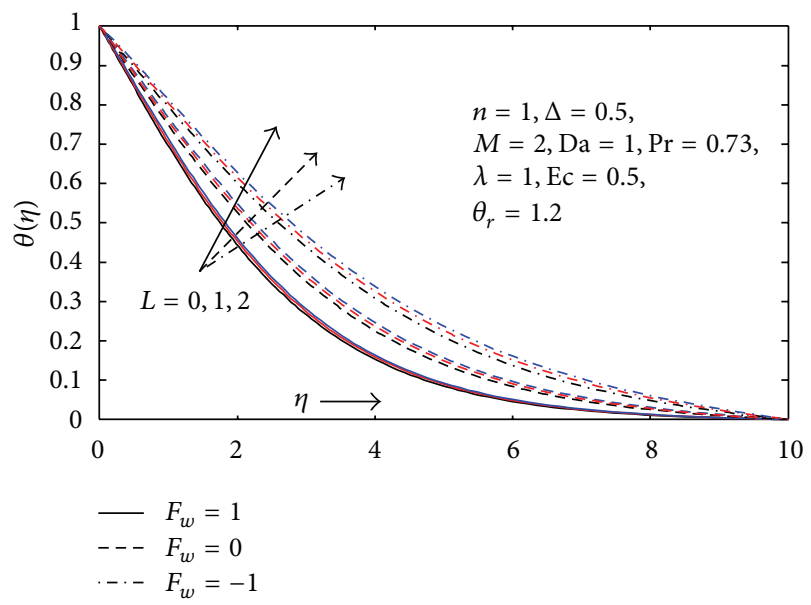

Figure 14: Temperature profile for different values of $F_{w}$ and $L$.

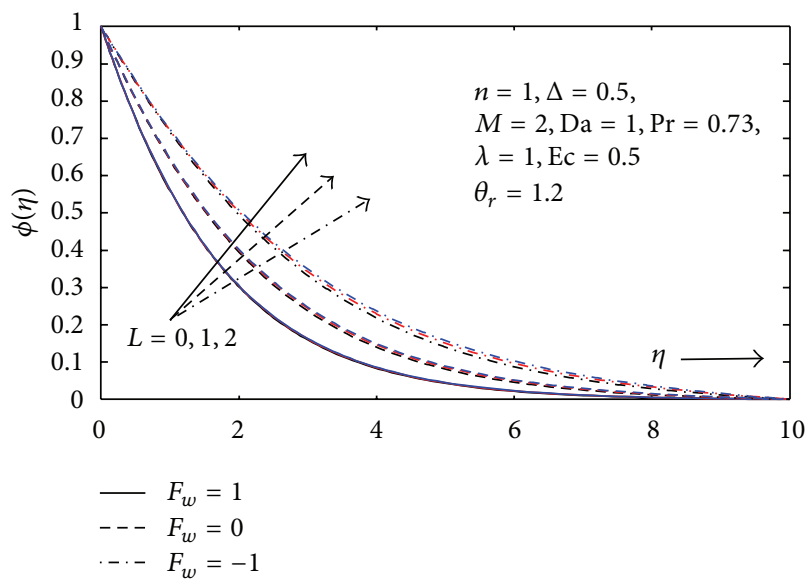

FiguRE 15: Concentration profile for different values of $F_{w}$ and $L$.

is due to extra mixing of fluid layers due to the enhanced shear stress. This is accompanied by a slight increase in the wall deposition flux. In addition, the effect of Sc on the local skin friction and local Nusselt number becomes more pronounced with increasing buoyancy force parameter $\lambda$, since the buoyancy force acts as a favorable pressure gradient. 


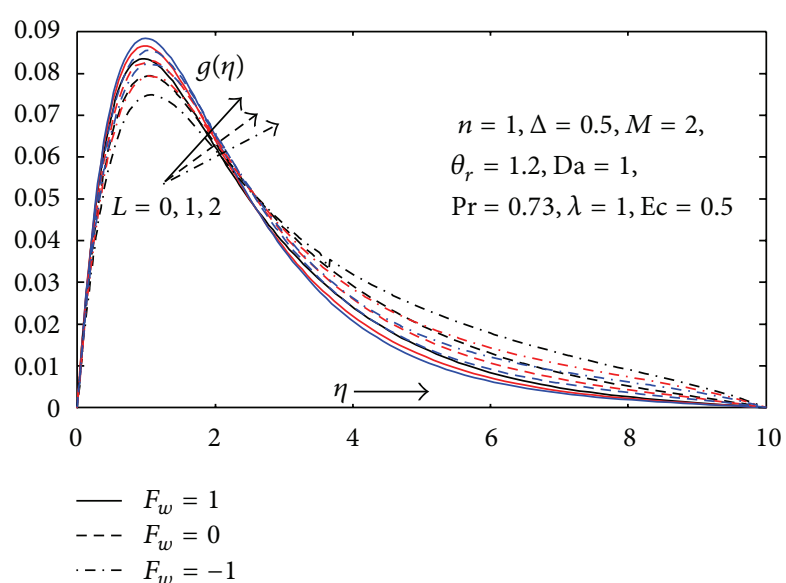

FIGURE 16: Angular velocity distribution for different values of $F_{w}$ and $L$.

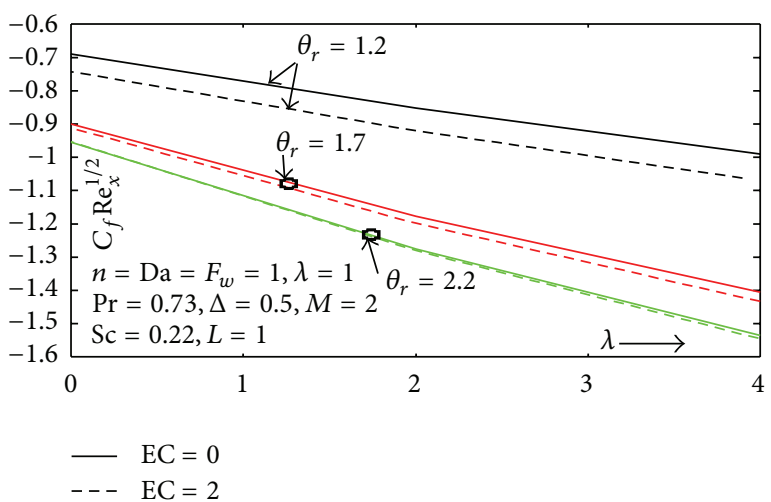

FIGURE 17: Local skin friction for different values of Ec and $\theta_{r}$.

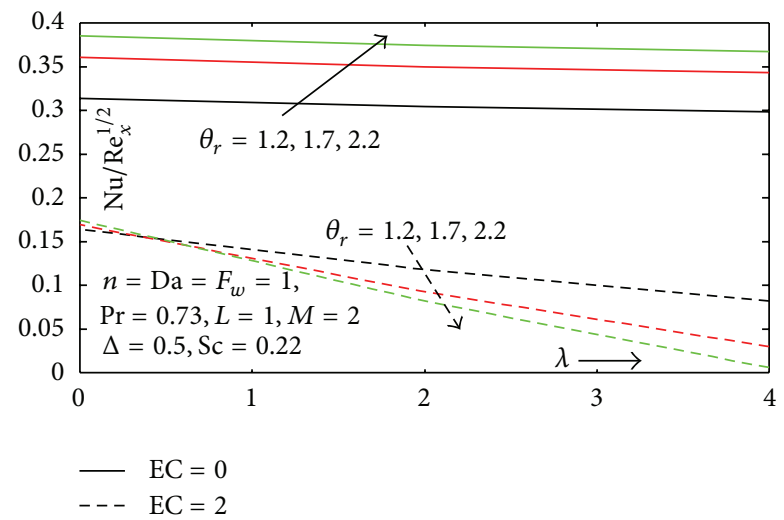

Figure 18: Local Nussselt number for different values of Ec and $\theta_{r}$.

\section{Conclusions}

A mathematical analysis has been carried out to study the MHD boundary layer flow of a micropolar fluid with medium molecular weight along a permeable stretching surface embedded in a non-Darcian porous medium with viscous dissipation and chemical reaction. The fluid viscosity

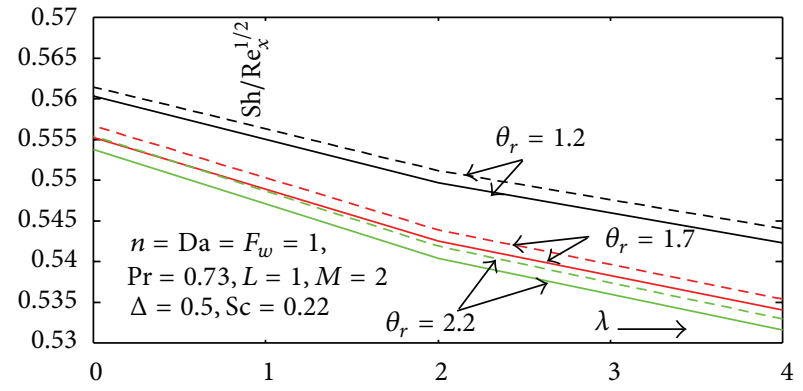

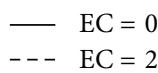

FIGURE 19: Local Sherwood number for different values of Ec and $\theta_{r}$.

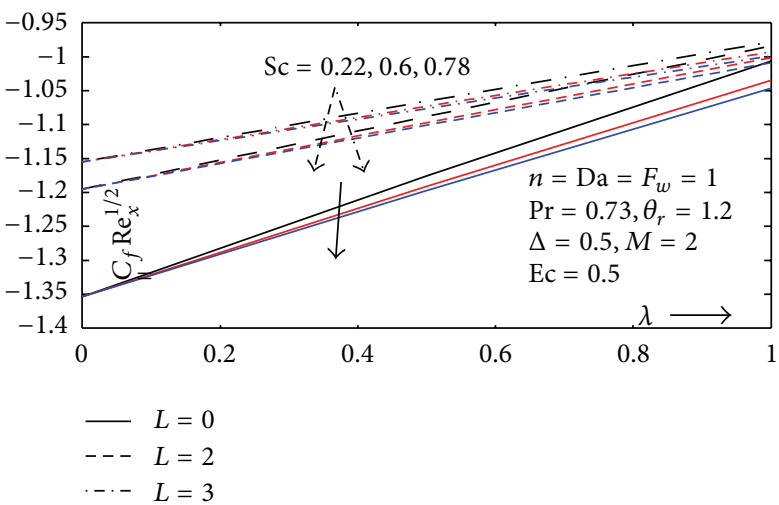

Figure 20: Local skin-friction for different values of Sc and $L$.

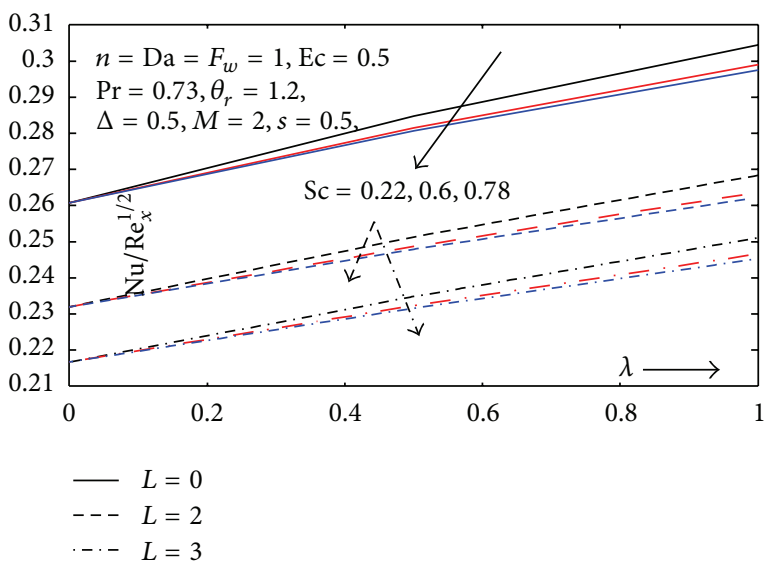

Figure 21: Local Nusselt number for different values of Sc and $L$.

is assumed to vary as an inverse linear function of temperature. The resulting partial differential equations, which describe the problem, are transformed into ordinary differential equations by using a similarity transformations and then solved numerically by shooting method. A comparison between the analytical and the numerical solutions has been included, and the results are found to be in excellent agreement. A representative set of numerical results for velocity, temperature and concentration profiles as well as the local skin-friction coefficient, the local Nusselt number, and local 


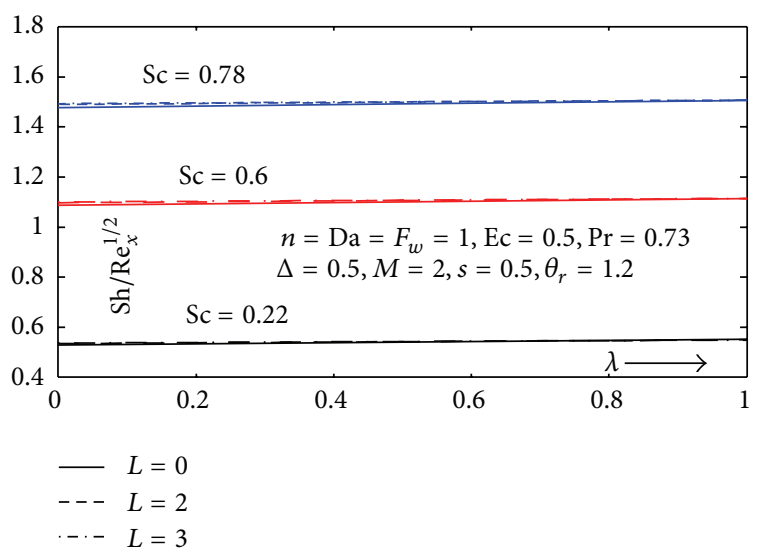

FIGURE 22: Local Sherwood number for differentvalues of Sc and $L$.

Sherwood number is presented graphically and discussed. It is found that the momentum boundary thickness as well as thermal boundary layer thickness decrease with increasing in the variable viscosity parameter $\theta_{r}$. It is of interest to note that the heat transfer strongly depends on the viscous dissipation Ec. In the presence of the latter, the effect of increasing values of $\theta_{r}$ is seen to increase the rate of heat transfer near surface and to decrease the same significantly away from the surface. This reduction of heat transfer away from the boundary would be more for large values of buoyancy force parameter $\lambda$. The ability of the microelements of the fluid to rotate decreases as the variable viscosity parameter $\theta_{r}$ increases, and it increases away from the plate due to the presence of viscous dissipation. The local friction coefficient is highly affected by the viscous dissipation for small values of $\theta_{r}$. However, for large $\theta_{r}$, the local friction coefficient is slightly influenced by the viscous dissipation effect. Therefore, we conclude that for a micropolar fluid of hydrogen-air mixtures, the variable viscosity and viscous dissipation effects should not be neglected. Increasing the coupling parameter tends to increase the local friction coefficient but tends to decrease the heat transfer rate and slightly increase the local Sherwood number. Increasing the Schmidt number tends to increase the local Sherwood number but tends to decrease the local friction coefficient. Finally, our numerical computations also indicate that the local skin-friction and the local Sherwood number are lower for the case of Newtonian fluids $(L=0)$ as compared with the micropolar fluids $(L>0)$. Also, the rate of heat transfer for the micropolar fluid is considerably less than that for the Newtonian fluid.

\section{References}

[1] A. C. Eringe, "Heat Simple micropolar fluids," International Journal of Engineering Science, vol. 2, pp. 205-217, 1964.

[2] A. C. Eringen, "Theory of micropolar fluids," vol. 16, pp. 1-18, 1966.

[3] A. C. Eringen, "Theory of thermomicrofluids," Journal of Mathematical Analysis and Applications, vol. 38, no. 2, pp. 480496, 1972.
[4] T. Ariman, M. A. Turk, and N. D. Sylvester, "Microcontinuum fluid mechanics-a review," International Journal of Engineering Science, vol. 11, no. 8, pp. 905-930, 1973.

[5] T. Ariman, M. A. Turk, and N. D. Sylvester, "Applications of microcontinuum fluid mechanics," International Journal of Engineering Science, vol. 12, no. 4, pp. 273-293, 1974.

[6] G. Łukaszewicz, Micropolar Fluids, Birkhäuser, Boston, Mass, USA, 1999, Theory and applications.

[7] A. C. Eringen, Microcontinum Field Theories, II. Fluent Media, Springer, New York, NY, USA, 2001.

[8] E. M. Abo-Eldahab and M. A. El Aziz, "Flow and heat transfer in a micropolar fluid past a stretching surface embedded in a nonDarcian porous medium with uniform free stream," Applied Mathematics and Computation, vol. 162, no. 2, pp. 881-899, 2005.

[9] N. T. Eldabe, A. G. El-Saka, and A. Fouad, “Thermal-diffusion and diffusion-thermo effects on mixed free-forced convection and mass transfer boundary layer flow for non-Newtonian fluid with temperature dependent viscosity," Applied Mathematics and Computation, vol. 152, no. 3, pp. 867-883, 2004.

[10] N. T. M. Eldabe and M. A. A. Mohamed, "Heat and mass transfer in hydromagnetic flow of the non-Newtonian fluid with heat source over an accelerating surface through a porous medium," Chaos, Solitons and Fractals, vol. 13, no. 4, pp. 907-917, 2002.

[11] M. Bourich, M. Hasnaoui, A. Amahmid, and M. Mamou, "Soret convection in a shallow porous cavity submitted to uniform fluxes of heat and mass," International Communications in Heat and Mass Transfer, vol. 31, no. 6, pp. 773-782, 2004.

[12] R. Bhargava, S. Sharma, H. S. Takhar, O. A. Beg, and P. Bhargava, "Numerical solutions for micropolar transport phenomena over a stretching sheet," Nonlinear Analysis: Modeling and Control, vol. 12, pp. 45-63, 2007.

[13] D. Pal and S. Chatterjee Sewli, "Mixed convection magnetohydrodynamic heat and mass transfer past a stretching surface in a micropolar fluid-saturated porous medium under the influence of Ohmic heating, Soret and Dufour effects," Communications in Nonlinear Science and Numerical Simulation, vol. 16, no. 3, pp. 1329-1346, 2011.

[14] U. N. Das, R. Deka, and V. M. Soundalgekar, "Effects of mass transfer on flow past an impulsively started infinite vertical plate with constant heat flux and chemical reaction," Forschung im Ingenieurwesen/Engineering Research, vol. 60, no. 10, pp. 284287, 1994.

[15] R. Kandasamy, K. Periasamy, and K. K. S. Prabhu, "Effects of chemical reaction, heat and mass transfer along a wedge with heat source and concentration in the presence of suction or injection," International Journal of Heat and Mass Transfer, vol. 48, no. 7, pp. 1388-1394, 2005.

[16] D. Pal and S. Chatterjee, "Heat and mass transfer in MHD non-Darcian flow of a micropolar fluid over a stretching sheet embedded in a porous media with non-uniform heat source and thermal radiation," Communications in Nonlinear Science and Numerical Simulation, vol. 15, no. 7, pp. 1843-1857, 2010.

[17] S. M. M. El-Kabeir and M. Modather, "Chemical reaction, heat and mass transfer on MHD flow over a vertical isothermal cone surface in micropolar fluids with heat generation/absorbtion," Applied Mathematical Sciences, vol. 34, pp. 1663-1674, 2007.

[18] A. A. Bakr, "Effects of chemical reaction on MHD free convection and mass transfer flow of a micropolar fluid with oscillatory plate velocity and constant heat source in a rotating frame of reference," Communications in Nonlinear Science and Numerical Simulation, vol. 16, no. 2, pp. 698-710, 2011. 
[19] H. Herwing and K. Gersten, "The effect of variable properties on laminar boundary layer flow," Warm Staffuberter, vol. 20, pp. 47-52, 1986.

[20] S. Mukhopadhyay, G. C. Layek, and S. A. Samad, "Study of MHD boundary layer flow over a heated stretching sheet with variable viscosity," International Journal of Heat and Mass Transfer, vol. 48, no. 21-22, pp. 4460-4466, 2005.

[21] S. Mukhopadhyay and G. C. Layek, "Effects of thermal radiation and variable fluid viscosity on free convective flow and heat transfer past a porous stretching surface," International Journal of Heat and Mass Transfer, vol. 51, no. 9-10, pp. 2167-2178, 2008.

[22] M. E. Ali, "The effect of variable viscosity on mixed convection heat transfer along a vertical moving surface," International Journal of Thermal Sciences, vol. 45, no. 1, pp. 60-69, 2006.

[23] O. D. Makinde, "Laminar falling liquid film with variable viscosity along an inclined heated plate," Applied Mathematics and Computation, vol. 175, no. 1, pp. 80-88, 2006.

[24] K. V. Prasad, K. Vajravelu, and P. S. Datti, "The effects of variable fluid properties on the hydro-magnetic flow and heat transfer over a non-linearly stretching sheet," International Journal of Thermal Sciences, vol. 49, no. 3, pp. 603-610, 2010.

[25] M. S. Alam, M. M. Rahman, and M. A. Sattar, "Transient magnetohydrodynamic free convective heat and mass transfer flow with thermophoresis past a radiate inclined permeable plate in the presence of variable chemical reaction and temperature dependent viscosity," Nonlinear Analysis: Modelling and Control, vol. 14, no. 1, pp. 3-20, 2009.

[26] A. M. Salem, "Variable viscosity and thermal conductivity effects on MHD flow and heat transfer in viscoelastic fluid over a stretching sheet," Physics Letters A, vol. 369, no. 4, pp. 315-322, 2007.

[27] M. A. Seddeek and F. A. Salama, "The effects of temperature dependent viscosity and thermal conductivity on unsteady MHD convective heat transfer past a semi-infinite vertical porous moving plate with variable suction," Computational Materials Science, vol. 40, no. 2, pp. 186-192, 2007.

[28] F. C. Lai and F. A. Kulacki, "The effect of variable viscosity on convective heat transfer along a vertical surface in a saturated porous medium," International Journal of Heat and Mass Transfer, vol. 33, no. 5, pp. 1028-1031, 1990.

[29] B. Gebhard, Y. Jaluria, R. L. Mahajan, and B. Sammakia, Buoyancy-Induced Flow and Transport, Hemisphere, New York, NY, USA, 1988. 


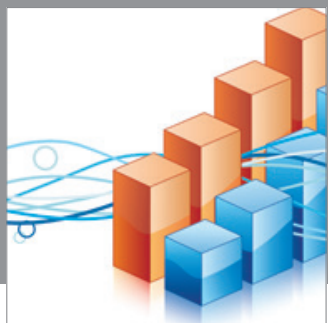

Advances in

Operations Research

mansans

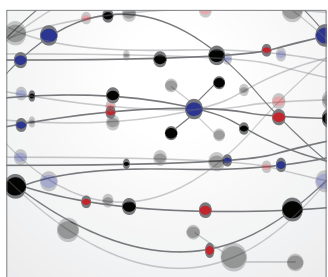

The Scientific World Journal
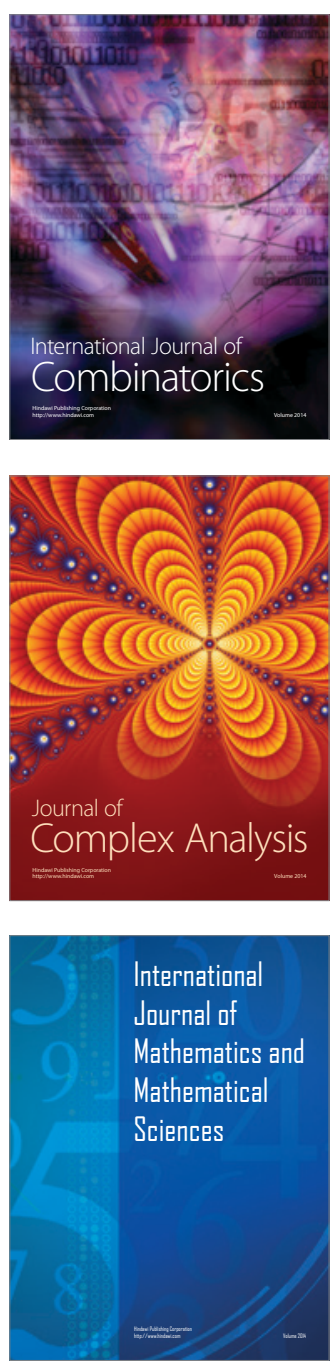
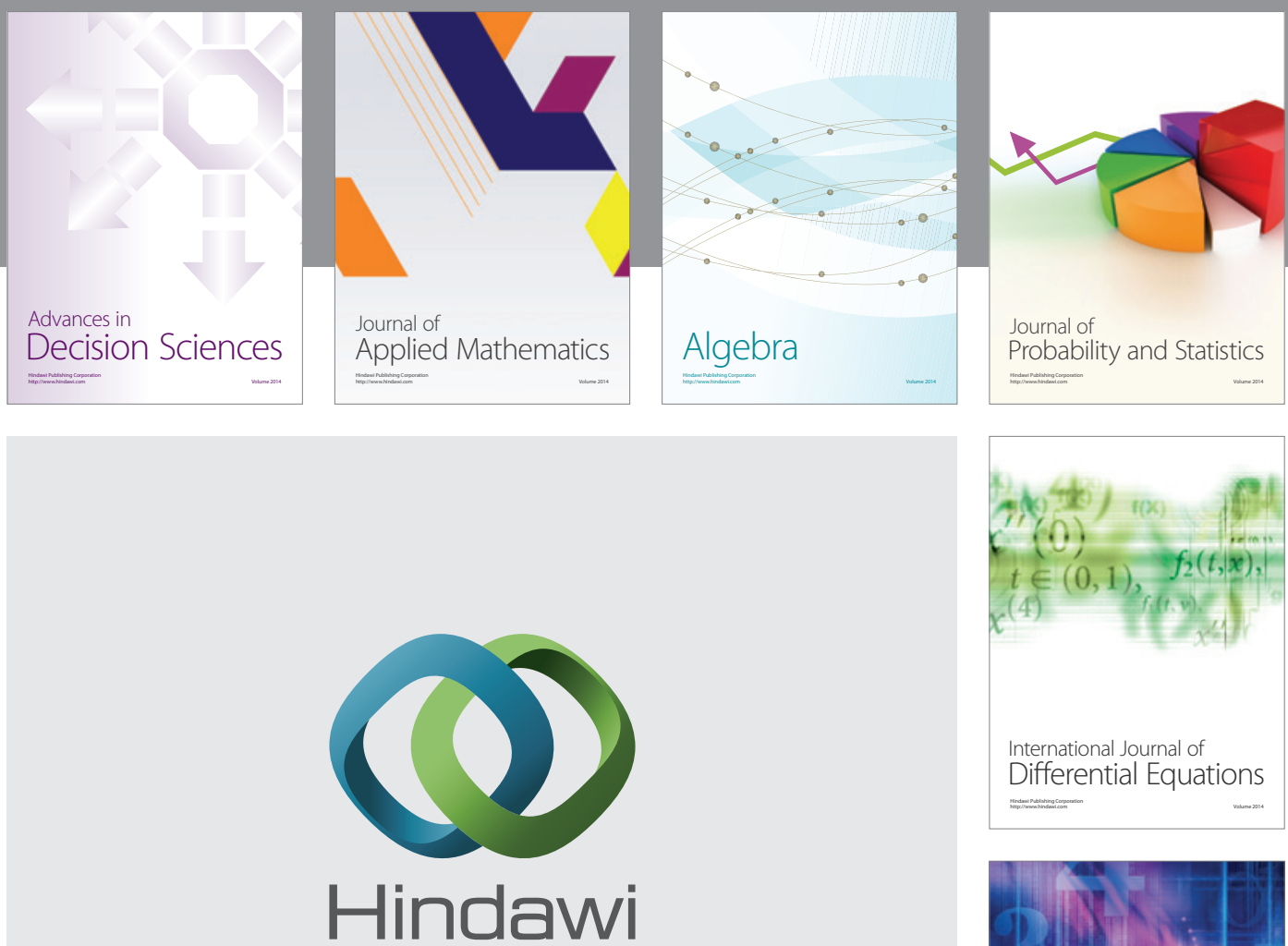

Submit your manuscripts at http://www.hindawi.com
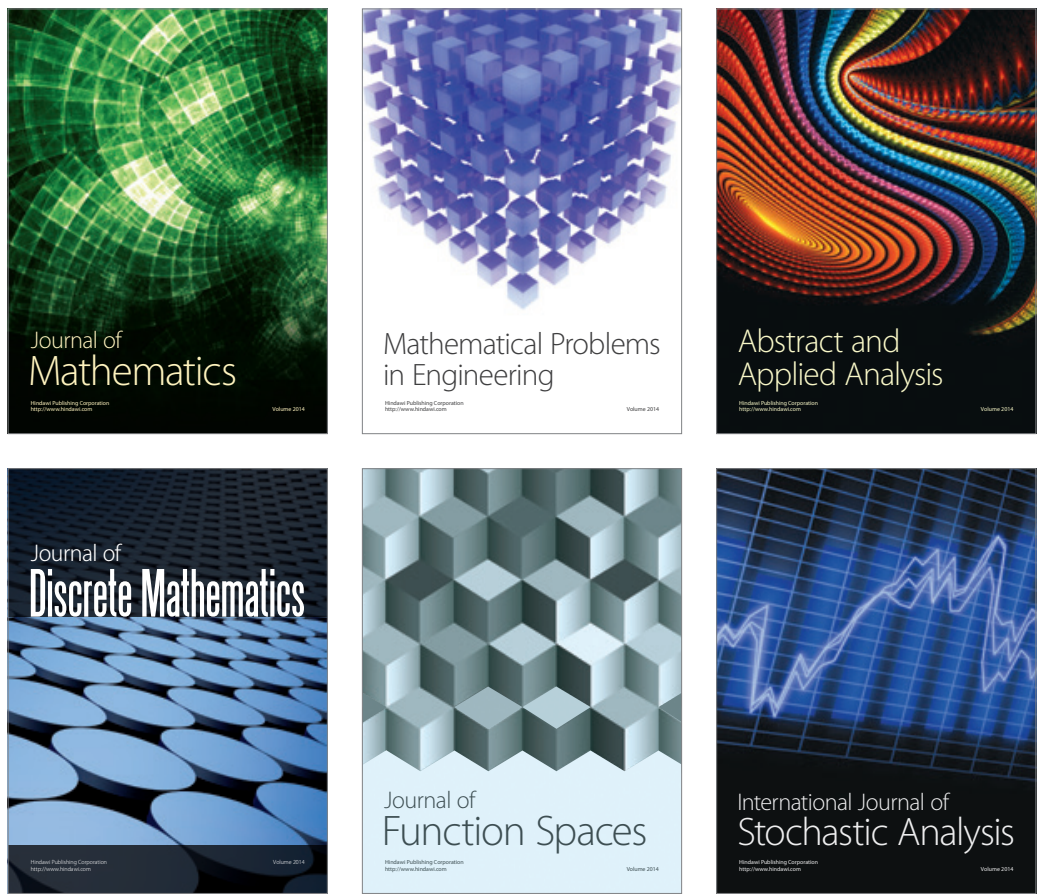

Journal of

Function Spaces

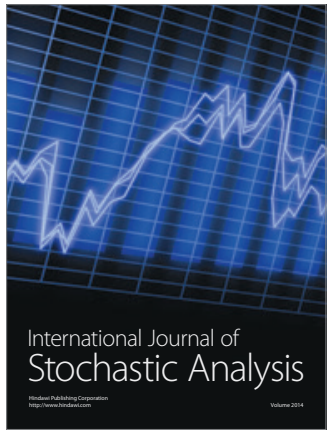

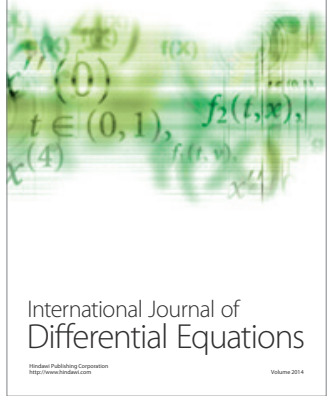
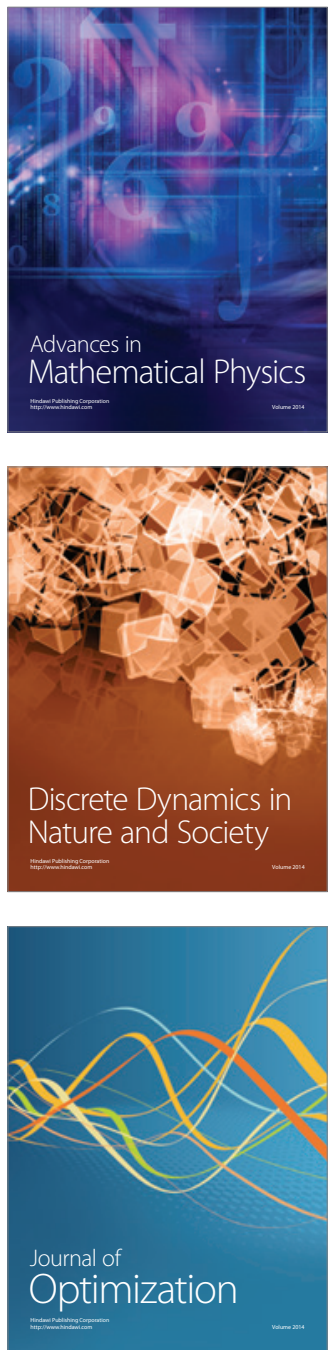